\title{
Juno reveals new insights into lo-related decameter radio emissions
}

Martos, Yasmina M.; Imai, Masafumi; Connerney, John E. P.; Kotsiaros, Stavros; Kurth, William S.

Published in:

Journal of Geophysical Research: Planets

Link to article, DOI:

10.1029/2020JE006415

Publication date:

2020

Document Version

Peer reviewed version

Link back to DTU Orbit

Citation (APA):

Martos, Y. M., Imai, M., Connerney, J. E. P., Kotsiaros, S., \& Kurth, W. S. (2020). Juno reveals new insights into lo-related decameter radio emissions. Journal of Geophysical Research: Planets, 125(7), [e2020JE006415]. https://doi.org/10.1029/2020JE006415

\section{General rights}

Copyright and moral rights for the publications made accessible in the public portal are retained by the authors and/or other copyright owners and it is a condition of accessing publications that users recognise and abide by the legal requirements associated with these rights.

- Users may download and print one copy of any publication from the public portal for the purpose of private study or research.

- You may not further distribute the material or use it for any profit-making activity or commercial gain

- You may freely distribute the URL identifying the publication in the public portal 
Martos Yasmina, M. (Orcid ID: 0000-0003-0351-7825)

Imai Masafumi (Orcid ID: 0000-0002-2814-4036)

Connerney John, E. P. (Orcid ID: 0000-0001-7478-6462)

Kotsiaros Stavros (Orcid ID: 0000-0003-2636-5545)

Kurth William, S (Orcid ID: 0000-0002-5471-6202)

\title{
Juno reveals new insights into Io-related decameter radio emissions
}

\section{Yasmina M. Martos ${ }^{1,2}$, Masafumi Imai ${ }^{3,4}$, John E.P. Connerney ${ }^{1,5}$, Stavros Kotsiaros ${ }^{6}$ and William S. Kurth ${ }^{3}$}

${ }^{1}$ NASA Goddard Space Flight Center, Greenbelt, MD, USA

${ }^{2}$ University of Maryland, College Park, MD, USA

${ }^{3}$ University of Iowa, Iowa City, Iowa, USA

${ }^{4}$ Department of Electrical Engineering and Information Science, National Institute of Technology (KOSEN), Niihama College, Niihama, Ehime, Japan

${ }^{5}$ Space Research Corporation, Annapolis, MD, USA

${ }^{6}$ Technical University of Denmark, Lyngby, Denmark

Corresponding author: Yasmina M. Martos (yasmina.martos@nasa.gov)

\section{Key Points:}

- Latest magnetic field model (JRM09) is used to compute Io-related decametric radio emissions and parameters associated with it

- Beaming angles range from $33^{\circ}$ to $85^{\circ}$ and estimated resonant electron energies are up to 23 times larger than proposed in the past

- The higher number of observations of groups of arcs originating in the northern hemisphere is a consequence of the magnetic field geometry

\begin{abstract}
The Juno mission is providing stunning new information about Jupiter and its environment. A new magnetic field model (JRM09) with much improved accuracy near the planet provides the basis for a better understanding of Io-related decametric radio emissions (DAM) and implications for auroral processes. Here, we selected Io-related DAM events observed by the Juno Waves instrument to shed light into the beaming angle, the resonant electron energy and radio source location by forward modeling. We use the JRM09 model to better constrain the location and observability of DAM, and characterize the loss cone-driven electron cyclotron maser instability. We obtained good agreement between synthetic and observed arcs with calculated beaming angles ranging from $33^{\circ}$ to $85^{\circ}$ and resonant electron energies up to 23

This article has been accepted for publication and undergone full peer review but has not been through the copyediting, typesetting, pagination and proofreading process which may lead to differences between this version and the Version of Record. Please cite this article as doi: 10.1029/2020JE006415
\end{abstract}


times higher than previously proposed. In addition, through a quantitative analysis, we provide an explanation regarding the higher likelihood of observing groups of arcs originating in the northern hemisphere relative to those originating in the southern hemisphere. This is primarily a consequence of the asymmetry of the magnetic field geometry, observer location, and pitch angles of the electrons at the equator.

\section{Plain Language Summary}

The interaction between Jupiter's magnetic field and Io has been observed from Earth via radio waves detection since 1955 . The radio waves are generated by energetic electrons traveling along Jupiter's magnetic field and are observed when the instrument, Io and the Jovian magnetic field are under particular geometric configurations. The radio waves may appear in groups and it has been observed that those originating in the northern hemisphere are more abundant than their southern hemisphere counterparts. However, no explanation for this observation was proposed until now. Thanks to Juno, the geometry of the magnetic field has been better constrained as waves and magnetic field data have been continuously collected within the Jovian environment since July 2016. In this study, we estimate where the radio waves generate and the energy of the electrons that generate these waves, which is up to 23 times higher than previously proposed. We ultimately demonstrate that the geometry of Jupiter's magnetic field is a primary controller for the higher observation likelihood of radio wave groups originating in the northern hemisphere relative to those originating in the southern hemisphere.

\section{Introduction}

Io-related decametric radio emissions (DAM) are the radio frequency expression of a complex electrodynamic interaction between Jupiter and its moon Io. Neutral particles lost by Io are rapidly ionized creating the plasma torus around Jupiter at Io's orbit [Thomas et al., 2004]. The new plasma is then dragged by Jupiter's magnetic field towards corotation and electrons are accelerated along magnetic field lines toward the planet causing aurorae and DAM emissions. These radio emissions range from 1 to $\sim 40 \mathrm{MHz}$ and have been observed from Earth since 1955 [Burke and Franklin, 1955] and from spacecraft during flybys [Genova and Aubier, 1985]. Since DAM is emitted in a narrow conical beam oriented by the magnetic field, the probability of detection depends on the geometrical configuration among the observer, Io and Jupiter. The Central Meridian Longitude (CML), defined in the Jovian System III coordinate system, describes the position of the observer. The Io Phase, $\Phi_{\mathrm{Io}}$, represents the angle between Io and the observer's antemeridian (Figure 1).

Based on the identification of events in the Io phase-CML plane, Io-related DAM emissions are typically divided in four groups: A, B, C and D [Carr et al., 1983; Marques et al., 2017]. Sources A and B originate in the northern hemisphere while C and D originate in the southern hemisphere. Sources B and D are known as vertex early events and present an open parenthesis arc shape in the time-frequency plane. They are observed when Io phase is near $90^{\circ}$. Sources $\mathrm{A}$ and $\mathrm{C}$, known as vertex late events, are characterized as closed parentheses and are observed near $\sim 240^{\circ}$ Io phase. 
Io-related DAM is thought to result from the cyclotron maser instability (CMI) [Wu and Lee, 1979; Treumann, 2006], in which unstable electron distributions convert into wave energy. Hence, the electrons are accelerated along or near the Io Flux Tube (IFT), the magnetic field lines connecting Io with Jupiter. DAM emissions originate above Jupiter's atmosphere at the local gyrofrequency along the magnetic field line carrying the active radio sources known as the Active Flux Tube (AFT). The lead angle is defined as the angle between the IFT and the AFT in the orbital plane. The least well-known parameters relating to Io-related DAM emissions are the lead angle, the energy of the resonant electrons and its beaming angle - the angle between the magnetic field vector at the radio source and the wave vector. This angle varies with the frequency of the emission and is related to a loss cone distribution producing oblique emission [Hess et al., 2008], recently confirmed by Kurth et al. [2017a] and Louarn et al. [2017]. The beaming angle is independent of the source region but it is strongly dependent on the geometry of the magnetic field. Many attempts to simulate Io-related arcs have been performed in the past [Queinnec and Zarka, 1998; Zarka, 1998; Clarke et al., 2004; Hess et al., 2008; Bagenal et al., 2017] considering the magnetic field models available at the time, such as the VIP4 [Connerney et al., 1998], GSFC O4 [Acuña and Ness, 1976a; 1976b], GSFC O6 [Connerney, 1992], VIPAL [Hess et al., 2011], constructed using magnetic field data acquired by the flybys of Voyager 1 and Pioneer 11. The VIP4 model also incorporated additional constraints on the field geometry from observations of the position of the IFT footprint; the VIPAL model added other constraints based on models of DAM radiation. These spherical harmonic models resolved the magnetic field geometry up to a maximum of degree $\leq 5$. However, early results from Juno's magnetic mapping mission provided a much more accurate magnetic field model, the JRM09 [Connerney et al., 2018], which characterizes the magnetic field up to spherical harmonic degree 10. A recent study using ExPRES tool (Exoplanetary and Planetary Radio Emission Simulators) has implemented the JRM09 magnetic field model to reproduce the arcs in the time-frequency plane [Louis et al., 2019].

The better understanding of the magnetic field geometry provided by Juno together with continuous measurements of radio emissions by the Waves instrument [Kurth et al, 2017b] provides an excellent opportunity to constrain the radio sources and parameters involved in the Io-related DAM emissions and their observability. Here, we study Io-related DAM events from each source location (A, B, C, D) identified by the Juno Waves Investigation since May 2016. We estimate the energy of the resonant electrons responsible for the wave-particle interactions, the beaming cone half-angles, and compare our results with previous studies. We also quantitatively explain why groups of arcs originating in the northern hemisphere are more frequently observed than those originating in the south.

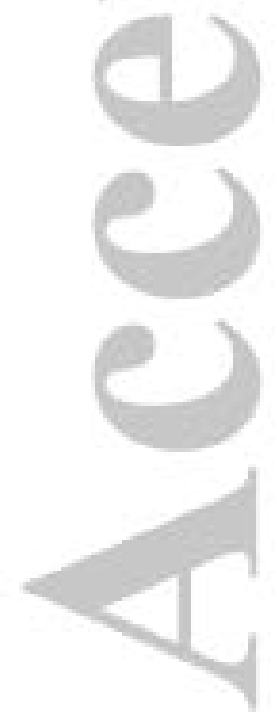




\section{Data and Methods}

Juno spacecraft has been in polar orbit about Jupiter since July 2016, recording observations with its nine onboard instruments [Bolton et al., 2017] including the magnetometer [Connerney et al., 2017a, 2017b] and the radio and plasma waves (Waves) instrument [Kurth et al., 2017b]. The Waves instrument measures Jupiter radio emissions at frequencies up to $41 \mathrm{MHz}$, beginning with the first detection of Jovian DAM made during Juno's interplanetary cruise in May 2016. Waves data are recorded by three onboard receivers that record electric fields from $50 \mathrm{~Hz}$ to $41 \mathrm{MHz}$. The receivers work on five different frequency bands; we use only the higher frequency band with 38 linearly spaced channels (3.5 $-40.5 \mathrm{MHz}$ ) and with a noise bandwidth of $666 \mathrm{kHz}$. The temporal resolution of the instrument is selectable at 1, 10 or $30 \mathrm{~s}$ depending on the operational mode, and we make use of all three options in our study. However, the continuously acquired signal at a frequency of $\sim 20 \mathrm{MHz}$ observed in the time-frequency spectrogram is heavily attenuated due to on-board interference associated with one of Juno's crystal oscillators (Figure 2).

2.1 Observations and modeling

\subsubsection{Observations}

We analyzed the Waves radio data in the time-frequency plane to detect well-defined discrete Io-related DAM events by visual survey. Accordingly, we selected four examples, one from each radio source location (Figure 2), to determine the lead angle (or the location of AFT in Io's orbit), the energy of the resonant electrons responsible for the radiation and the beaming cone half-angle of the emissions.

The spectral data for these events is presented in time-frequency diagrams (Figure 2). The selected vertex early events were observed on October 17 and October 3 of 2017. The Io phase and Jovian CML at the time of observation clearly identify these emissions as originating from the B and D source regions, respectively. The B source emission is characterized by a group of four clearly defined discrete events (Figure 2a) with frequencies that extend up to 32 $\mathrm{MHz}$ and are observed over $45 \mathrm{~min}$. The D source emission (Figure $2 \mathrm{~b}$ ) is composed of a single discrete arc lasting for about $90 \mathrm{~min}$ and reaching a peak frequency of $16 \mathrm{MHz}$.

Based on the relative positions of Juno, Jupiter and Io, the vertex late emissions observed on July 15 and March 12 of 2018 correspond to A and C radio source regions, respectively. The A radio source emission (Figure 2c) is composed of a group of three welldefined discrete arcs observed for $\sim 40 \mathrm{~min}$ and comprising peak frequencies between 28 and $37 \mathrm{MHz}$. A single discrete arc characterizes the $\mathrm{C}$ emission (Figure $2 \mathrm{~d}$ ) reaching $20 \mathrm{MHz}$ as a peak frequency and lasting about $40 \mathrm{~min}$.

In this study, the events corresponding to radio sources originating in the northern hemisphere (A and $\mathrm{B}$ ) reach much higher frequencies than those originating in the southern hemisphere ( $C$ and $D)$. Additionally, the southern hemisphere emissions are characterized by a single discrete and very curved arc in a time-frequency spectrogram while the northern ones are composed by several discrete events.

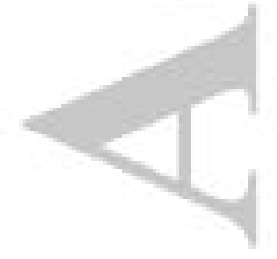




\subsubsection{Modeling}

In the context of CMI theory, the relativistic framework for the resonant condition as well as an unstable electron velocity distribution (e.g. loss cone, ring shell) are related to the beaming angle. If we assume the theoretical treatment of a relationship between the cone half-angle and the loss cone electron distribution proposed by Hess et al. [2008] (eq. 1), we can estimate the energy of the resonant electron once the beaming angle is determined from analysis of the geometry of observation. The Io-related DAM events are computed via forward modeling using the following relation:

$$
\theta(f)=\arccos \left[\frac{v_{e}}{c N} \frac{1}{\sqrt{1-f / f_{\max }}}\right]
$$

where $f$ is the observed frequency assumed to be the local gyrofrequency at the radio source, $f_{\max }$ is the maximum gyrofrequency reached at the foot of the AFT (or Jupiter's surface, 1 bar level), $c$ is the speed of light, $N$ is the refraction index and $v_{e}$ is the velocity of the resonant electron, linked to its energy by $E_{e}=m_{e} v_{e}^{2} / 2$ with $m_{e}$ as the electron mass.

Forward modeling of the discrete arcs observed in the spectrograms is carried out considering the geometrical configuration of the fixed thin hollow cone at the radio source and the loss cone-driven CMI theory described above as follows:

a) The radio sources are located along the AFT, the magnetic field line that links Io's orbit $\left(\mathrm{R}=5.9 \mathrm{R}_{\mathrm{J}}\right.$, Jupiter radii) with Jupiter.

b) The radio wave is assumed to propagate in a straight line from the source to the Juno spacecraft.

c) The refraction index of the radio source environment is assumed to be 1 [Ray and Hess, 2008].

d) The location of the radio sources (Jovian longitude, latitude and radial distance) and the cone half-angle are estimated using the magnetic vector field computed using the JRM09 magnetic field model [Connerney et al., 2018] including the Jovian current sheet contribution [Connerney et al., 1981].

e) The spatial position of the observer is set at Juno's location.

f) The hollow cone of $1^{\circ}$ wall thickness [Kaiser et al., 2000; Panchenko and Rucker, 2016] is fixed at the location of the radio source.

g) When the geometry between the radio source and the observer is determined, the velocity of the resonant electron is calculated making use of equation (1) through a least squares fitting.

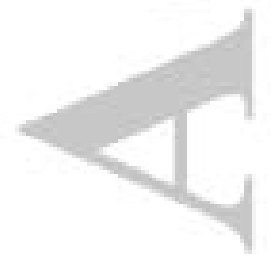


h) The resonant electron velocity obtained in g) is then used to compute the forward modeling of the cone half-angle for each observed frequencies of the discrete arc with the help of equation (1). Results were deemed satisfactory when the beaming cone half-angle determined by the geometrical constraint (configuration of Juno and the radio source) and the one obtained by forward modeling were comparable, reproducing the observed spectral arcs.

\section{Results: Beaming cone half-angle and resonant electron energy}

We found the best fit to the observations as well as the AFT, lead angle, resonant electron energy and the beaming cone half-angle. The southern hemisphere emissions ( $\mathrm{C}$ and D) were observed when the spacecraft was at large radial distances (105-110 $\mathrm{RJ}_{\mathrm{J}}$ ) and southern latitudes. A and B events were observed at closer radial distances and north of the equator.

The B radio source events (Figure 2a) originate at AFTs between $190^{\circ}$ to $211^{\circ}$ System III longitudes (at Io's orbit distance), corresponding to lead angles of $3^{\circ}-6^{\circ}$. The energy of the resonant electrons responsible for these emissions is between $1.6 \mathrm{keV}$ and $4.4 \mathrm{keV}$, and the estimated beaming angles range from $77^{\circ}$ to $85^{\circ}$. The peak frequencies of the arcs are located in the respective AFT at radial distances between $1.05 \mathrm{R}_{\mathrm{J}}$ and $1.09 \mathrm{R}_{\mathrm{J}}$.

The next modeled vertex early radio emission corresponds to a D source (Figure $2 b$ ). The lead angle and the resonant electron energy are much higher in this case reaching $40^{\circ}$ and $50 \mathrm{keV}$, respectively. The AFT responsible for this particular emission is $66^{\circ}$. The modeling yields beaming angles from $33^{\circ}$ to $60^{\circ}$. The closest radio source to Jupiter's atmosphere of this Io-related DAM emission is located at $1.07 \mathrm{R}_{\mathrm{J}}$.

The best fit for the arcs corresponding to the A radio source events (Figure 2c) is found for AFTs between $189^{\circ}$ and $196^{\circ}$. The resonant electron energies are also higher than those of the $\mathrm{B}$ events, ranging between 10 and $14 \mathrm{keV}$, while the lead angle $\left(1^{\circ}\right)$ and the cone half-angle $\left(65^{\circ}-77^{\circ}\right)$ are smaller. The highest frequencies originated within sources located between 1.04 and $1.07 \mathrm{R}_{\mathrm{J}}$.

The discrete arc from the $\mathrm{C}$ source region (Figure $2 \mathrm{~d}$ ) is best modeled with energies for the resonant electrons of $11 \mathrm{keV}$ at the AFT of $295^{\circ}$, which corresponds to a lead angle of $5^{\circ}$. The beaming cone angle is between $67^{\circ}$ and $76^{\circ}$. The peak frequency originated within a source at $1.04 \mathrm{R}_{\mathrm{J}}$.

The synthetic arcs fit the observations very well for the $\mathrm{B}$ and $\mathrm{C}$ arcs, with an error $\leq 1^{\circ}$. The A emissions and the low frequencies of the $\mathrm{D}$ arc are fit slightly less well, with errors $\leq 2^{\circ}$ and $\leq 3^{\circ}$, respectively. In this case, the errors are quantified using the difference between the beaming angle obtained by equation (1) and that obtained by examination of the geometrical configuration among the radio source, the magnetic field line and the spacecraft. 


\section{Discussion}

The Io-related DAM events carefully selected in this study show clear single discrete arcs or groups of arcs originating from each DAM source region. The observation of the emissions is geometrically constrained by the position of the spacecraft, Jupiter and Io, and modeled considering the loss cone driven CMI theory with the help of a specific Jovian magnetic field geometry. The JRM09 magnetic field model [Connerney et al., 2018] including the Jovian Current Sheet contribution [Connerney et al., 1981] has been used for line tracing and geometry of the AFT, leading to new results involving the origin of DAM. In addition to the dipolar asymmetry of the Jovian magnetic field, this model predicts much higher values at Jupiter's surface than previous models, and has a significant impact on the location of the sources, the beaming cone half-angle and the energy of the resonant electrons responsible for the emissions.

The range of beaming cone half-angle depends on the observation geometry, the resonant electron energy distribution, the magnetic field geometry, the local frequency and the maximum frequency at the foot of the active magnetic field line, which, in turn, depends on the magnetic field model. If we compare the possible beaming angles derived after using the peak frequencies at the Io foot print predicted by the JRM09 and the VIP4 models (Figure 3) we see that no realistic solutions are yielded for resonant electron energies above $40 \mathrm{keV}$ $\left(\mathrm{V}_{\mathrm{e}}=0.4 \mathrm{c}\right)$ and for local frequencies higher than $36 \mathrm{MHz}$ using VIP4. The solutions for both models are closer for low frequencies and low electron energies, while they differ the most for local frequencies above $20 \mathrm{MHz}$. For example, for observed frequencies of $35 \mathrm{MHz}$ and 23 $\mathrm{keV}$ resonant electron energy, we obtain a difference of more than $10^{\circ}$ for the beaming angle depending on the selected magnetic field model.

Forward modeling of the arcs yields new insights about the parameters involved in the emissions and location of the radio sources. The synthetic arcs delineate the observations very accurately (Figure 2 ) with errors between $<1^{\circ}$ and rarely $3^{\circ}$. Higher beaming cone half-angles are associated with the lower frequencies for the same discrete DAM arc. The vertex late events (A \& C) originate closer to the planet $\left(1.04 \mathrm{R}_{\mathrm{J}}\right)$ than vertex early events $\left(\sim 1.07 \mathrm{R}_{\mathrm{J}}\right)$. The energy of the resonant electron was higher for the $\mathrm{D}$ event $(50 \mathrm{keV})$ and lower for the $\mathrm{B}(1.6-4.4$ $\mathrm{keV}$ ), while for $\mathrm{A}$ and $\mathrm{C}$ was similar $(10-15 \mathrm{keV})$. Highest beaming angles $\left(77^{\circ}-85^{\circ}\right)$ were determined for the $\mathrm{B}$ radio sources (Figure $2 \mathrm{a}$ ), while the widest range corresponds to the $\mathrm{D}$ source $\left(33^{\circ}-60^{\circ}\right)$. Beaming angles for the vertex late emissions were comparable $\left(65^{\circ}-77^{\circ}\right)$.

The AFTs for the DAM events originating in the northern hemisphere correspond to magnetic field lines located at longitudes between $189^{\circ}$ and $211^{\circ}$ in Io's orbit and projected to $177^{\circ}-189^{\circ}$ System III longitude onto Jupiter's northern atmosphere (Figure 2). The AFT carrying the sources responsible for the $\mathrm{C}$ and $\mathrm{D}$ events are $295^{\circ}$ and $66^{\circ}$ respectively, which are projected to $311^{\circ}$ and $63^{\circ}$ System III longitude on the southern atmosphere of Jupiter. The footprint of the AFTs responsible for the northern hemisphere emissions intersects with the region with the highest magnetic field on the surface of the planet, between 18 and $20 \mathrm{G}$ [Connerney et al., 2018]. The footprint for the AFTs of the southern events also agrees with the highest magnetic field areas in the Jovian's southern hemisphere ( 14 G).

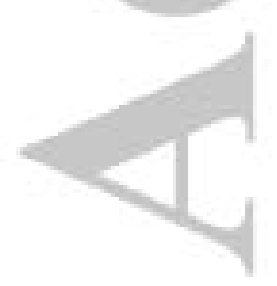


We acknowledge that the values obtained for the AFT and the energy of the resonant electron may not be unique. Substantial agreement with other studies is obtained regarding the AFTs carrying the radio sources in the northern hemisphere of Jupiter. Imai et al. [2017] described two non-Io-related discrete arcs observed by Juno and estimated the AFTs at $173^{\circ}$ and $176^{\circ}$ in System III. Also, Imai et al. [2008, 2011] localized radio sources in longitude ranges of $180^{\circ} \pm 10^{\circ}$ on Jupiter after modeling non-Io-A and B DAM sources observed by the Jupiter flyby of Cassini. The lead angles estimated by Hess et al. [2008] and Queinnec and Zarka [1998], like our results, range between few degrees and $40^{\circ}$. The location of these AFTs on Jupiter's surface (Figure 2, insets) as well as the ones that we propose for the selected A and $B$ events fall into the same high magnetic field area of the surface of Jupiter in the northern hemisphere [Connerney et al., 2018].

Our estimates for the energy of the resonant electrons responsible for the DAM emissions show generally higher values for the southern hemisphere than for the northern hemisphere. This is in agreement with the higher field aligned currents identified in the southern hemisphere [Kotsiaros et al., 2019] and with the pattern found in previous studies [Hess et al., 2008; Louis et al., 2017]. However, they differ significantly in its absolute values. We obtained up to $50 \mathrm{keV}$ for the resonant electron energy for the D event while other authors estimate $3 \mathrm{keV}$ [Hess et al., 2008; Louis et al., 2017] or $8 \mathrm{keV}$ [Louis et al., 2019]. A similar situation is found for the northern hemisphere where we estimate electron energies of up to 15 $\mathrm{keV}$ whereas an energy of $0.64 \mathrm{keV}$ was proposed in the past [e.g., Hess et al., 2008; Louis et al., 2017]. These differences are likely due to three main reasons: 1) the magnetic field model selected for the forward modeling, 2) the modeling procedure, and 3) the simple sine function used to fix the lead angle [Hess et al., 2011]. This simple sine estimate is possibly a poor approximation that does not consider the recent predictions for the main Alfven wing using JRM09 and recent plasma models [Hinton et al., 2019].

The lack of agreement between the values of the beaming cone half-angle for the Iorelated DAM relative to previous studies is due to the estimated velocity distributions of the resonant electrons and the use of a more accurate magnetic field model. In any case, we produce better fits between our models and the observations than other recent studies [Louis et al., 2017] wherein the authors obtained models that yield arcs around 1-2 $\mathrm{h}$ removed from the observation times as well as $\mathrm{MHz}$ of difference in frequency. 
In situ particle observations made by Juno's JADE (Jovian Auroral Distributions Experiment [McComas et al., 2017]) during Io footprint tail crossings during perijoves (closest approaches to Jupiter) 5, 6 and 7 [Szalay et al., 2018] detected downward electrons energy fluxes of $\sim 70 \mathrm{~mW} / \mathrm{m}^{2}$ near the Io footprint and $\sim 10 \mathrm{~mW} / \mathrm{m}^{2}$ farther in the tail. The electron energies measured in-situ are compatible with our estimates for the resonant electrons in our models. The Juno JADE and JEDI (Jupiter Energetic particle Detector Instrument, [Mauk et al., 2017]) observations for main aurora oval crossings have detected an important downward electron density for fluxes between 0.1 to $54 \mathrm{~mW} / \mathrm{m}^{2}$ and energy range of 10-50 keV [Ebert et al., 2019], also consistent with our estimates.

We can consider that our forward modeling of the arcs fit the measured discrete arcs and other observations nicely. However, especially for the low frequencies, improvement may be made considering: a) an electron velocity distribution instead a unique value for the entire arc, for which future observations of the JADE and JEDI instruments during close Io footprint crossing will be crucial, b) near source refraction close to the generation location of the radio sources [Galopeau and Boudjada, 2016], c) multiple AFT responsible for the emissions belonging to a single arc [Wilkinson, 1989], d) refraction index different than 1 for wave propagation. An extended Juno mission with orbits penetrating to lower altitudes at northern mid-latitudes may be expected to provide further insight into the generation of DAM radiation as well as the physics that drives DAM emissions.

\subsection{Observation of discrete arcs vs group of arcs}

Groups of arcs originating in the northern hemisphere (e.g., Figures $2 \mathrm{a}$ and $2 \mathrm{c}$ ) are much more commonly observed than those originating in the southern hemisphere, from which single discrete arcs are more often observed (e.g., Figures $2 b$ and $2 d$ ). Here, we provide several explanations for this fact relating the magnetic field geometry and intensity, Alfvén wave and particle propagation from Io to Jupiter, and observation geometry.

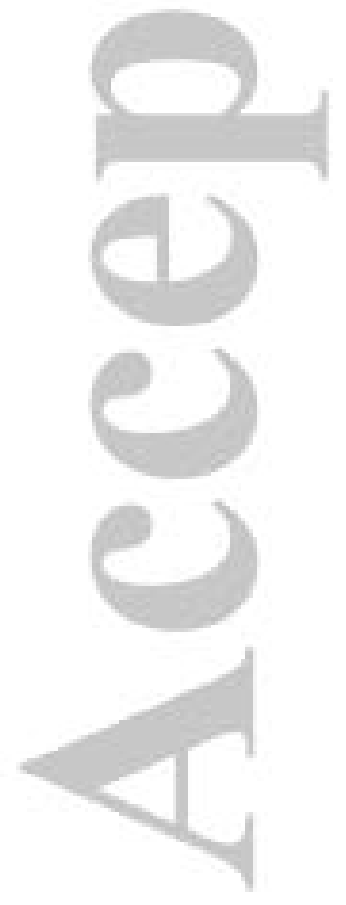


Figure 4 (panels $\mathrm{a}$ and $\mathrm{b}$ ) illustrates the relationship between pitch angle and Io longitude, assuming that the electrons traveling along the AFTs follow motions described by the first adiabatic invariant, $\sin ^{2} \alpha_{0}=\mathrm{B}_{0} / \mathrm{B}_{\max }$, where $\alpha_{0}$ and $\mathrm{B}_{0}$ are the pitch angle and the magnetic field strength at the Jovicentric equator, and $\mathrm{B}_{\max }$ is the magnetic field strength at the mirror point of the charged particle [Gurnett and Bhattacharjee, 2005]. For the purpose of this figure and in the case of Io-DAM, the mirror points are on the Io footprint and the pitch angles are computed at the equator using JRM09. Note that particles entering the Jovian magnetic field at Io's orbit with pitch angles less than $\sim 2.9-2.4^{\circ}$ and mirroring in the northern hemisphere (for example causing the selected A and B arcs in this study and represented by grey dots in Figure 4a) will precipitate in the southern hemisphere. Io-DAM arcs are believed to originate close to the planet by mirrored particles traveling away from Jupiter, although if loss cone conditions exist for downward electrons, DAM could also occur [Louarn et al., 2018]. As in almost $2 / 3$ of the Jovian longitudes the geometry and strength of the magnetic field in the northern hemisphere allows for lower values of pitch angles (Figure 4a), a great number of mirrored particles in the northern hemisphere precipitate in the southern hemisphere preventing Io-DAM from originating in the south at these longitudes. This will always be the case for Io longitudes of $70-295^{\circ}$. The higher precipitation of particles in the southern hemisphere is also supported by the more intense aurora observed by the Hubble Space telescope [Bonfond et al., 2013] and the Ultraviolet Imaging Spectrometer aboard Juno [Hue et al., 2019]. Then, based on Figure $4 \mathrm{a}$ and from the longitude point of view, the percentage of mirrored events for the northern hemisphere is higher than for the southern hemisphere, providing a plausible explanation for why more groups of arcs originate in the north relative to the south.

Since the geometry between observer, Jupiter, Io and the location of the radio source in the AFT dictates observability of DAM, we calculated and compared beaming cone half-angles considering two scenarios: 1) as a function of the local gyrofrequency and based on the relation proposed by Hess et al. [2008], also used to model the DAMs in our study (Figure 4c) and, 2) derived assuming a fixed geometry between the observer and Io and letting Jupiter rotate to explore the influence of the geometry of the magnetic field lines on the observability probability (Figure 5). We perform both scenarios for all Jovian longitudes and for the north and south hemispheres, assuming a vertex late configuration.

For scenario 1) we calculate the beaming cone half-angle at radio sources using equation 1, a resonant electron energy of $10 \mathrm{keV}$ (in agreement with our results for vertex late arcs) and different values for the local gyrofrequencies (Figure 4c). The beaming cone halfangle distribution shows that quasi-constant values are obtained in the northern hemisphere for AFTs centered in $\sim 180^{\circ}$. This behavior is not observed for the southern hemisphere. The magnetic field model predicts that this 'plateau' of beaming cone half-angle values in the northern hemisphere can be found for a large portion of AFTs (or Jovian longitudes); for example, between $120^{\circ}$ and $250^{\circ}$ for local gyrofrequencies of $20 \mathrm{MHz}$. But this situation is rarely the case for the southern hemisphere. In this case, this means that for an observer adequately located at $\sim 75.5^{\circ}$ of beaming cone half-angle for the emission of a specific radio source, an observer would be able to measure the specific frequency of $20 \mathrm{MHz}$ for about $130^{\circ}$ of Jovian rotation. This value is in reality higher since the wall thickness of the cone of emission is $1^{\circ}$ and the observer is able to measure arcs if these are located within the wall.

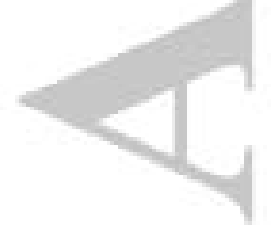


For scenario 2) we considered a fixed geometry between the observer and Io in a vertex late configuration with a rotating Jupiter to evaluate the influence of the geometry of the Jovian magnetic field in the observability of DAM. As DAM have been detected from Earth since 1955, we selected Earth as an observer located at $5 \mathrm{AU}$. A local gyrofrequency of $20 \mathrm{MHz}$ was selected to locate the radio sources in each AFT (or Jovian longitudes) to compute the geometrical beaming cone half-angle. The geometrical scenarios considered for both hemispheres also include the changes in Earth's latitude with respect to Jupiter $\left( \pm 3^{\circ}\right)$ and different Io phases $\left(240^{\circ}\right.$ and $\left.270^{\circ}\right)$ within a vertex late configuration (Figure 5). For DAM originating in the northern hemisphere, higher beaming cone half-angles are yielded when Earth is located at $-3^{\circ}$ of latitude, while for events originating in the southern hemisphere higher values are found for Earth located at $3^{\circ}$ of latitude. Our purpose with this scenario is to highlight that the northern hemisphere generally shows less variable beaming cone half-angles from one AFT (or Jovian longitude) to the next than the southern hemisphere. Notice that the long-term ground-based Io-DAM observations provide high occurrences where $\lambda_{\text {III }}$ ranges from $200^{\circ}$ through $20^{\circ}$ via $360^{\circ}$ at $205^{\circ}-260^{\circ}$ Io phases [e.g. Carr et al., 1983]. Using the equation of Io longitude $=\lambda \mathrm{III}+\left(180^{\circ}-\Phi_{\mathrm{Io}}\right)$ (see Figure 1$)$, this range is between $120^{\circ}$ and $355^{\circ}$ Io longitudes Consequently, the asymmetry of the geometry of the Jovian magnetic field influences the observation itself since the same frequency $(20 \mathrm{MHz})$ originating in the northern hemisphere can be measured for a larger number of AFT (or Jovian longitudes) while Jupiter rotates than for the southern hemisphere.

Scenarios 1) and 2) together with the pitch angle distribution provide quantitative explanations for the observation of higher number of groups of arcs originating in the north than in the south as a consequence of the asymmetry in the geometry of the Jovian magnetic field.

Regarding the traveling times of the Alfvén waves from Io to Jupiter, Hinton et al. [2019] predicted shorter times $(2.5 \mathrm{~min})$ for the north than the south $(\sim 11 \mathrm{~min})$ at longitudes of $\sim 190^{\circ}$ of System III. This region of magnetic field lines is in agreement with the AFTs determined for the modeled A and B arcs in this study. Faster traveling times will lead to faster activation of neighboring AFTs carrying DAM sources that can be viewed by the observer, which is, for a specific period of time, in the right geometry within the $1^{\circ}$ wall thickness of the hollow cone of emission. In addition, considering reflection of the same Alfvén wave within the Io plasma torus, Gurnett and Goertz [1981] estimated that the longitudinal separation between successive reflections was $5.8^{\circ}$ and the temporal separation was $20 \mathrm{~min}$ in average. These numbers are within the range of the modelled arcs in the northern hemisphere here. The separation of the Alfvén wave reflections is also larger in the southern hemisphere than in the northern when assuming Io located at $180^{\circ}$ of west longitude [Hinton et al., 2019]. The equally spaced peaks in the $\mathrm{H}_{3}^{+}$on Jupiter's atmosphere observed by Juno [Mura et al., 2018] and from Earth [Connerney and Satoh, 2000] $\left(\sim 5^{\circ}\right)$ also point to the multiple reflections of Alfvén waves within the torus and are indicator of the Io interaction and Jupiter's magnetosphere. If the Alfvén waves are responsible for accelerating the electrons that produce the DAM, a single Alfvén wave (and its reflections) that propagates out of the torus towards the planet can explain why the probability of observing groups of arcs that originate in the north is higher than for the south due to time travel and geometry of the observer with respect to the AFTs.

Summarizing, the combination of: a) pitch angles at the equator, b) location of the mirror points, c) asymmetry in the geometry of the Jovian magnetic field, d) multiple reflections and travel time of the Alfvén waves coming from the Io-plasma torus, and e) the position of the observer in the adequate location accounting for the beaming cone half-angle of DAM originating in the AFTs and the corotation of the magnetic field, provide the explanation 
regarding the more common observation of groups of arcs originating in the northern hemisphere than in the southern. In this aspect, an extended Juno mission will allow us to investigate this phenomenon as additional observational geometries will be explored.

\section{Conclusions}

In this study we selected main Io-DAM events, one from each source location, and made use of the most recent Jovian magnetic field model to model the observed discrete arcs. The forward modeling yielded very good fits to the observations and constrained the parameters, such as the resonant electron energy and the beaming cone half-angle, providing new insights into Jovian auroral radio emissions. The beaming angle is larger for the B events than the others and the AFTs projected on the surface of Jupiter coincide with the high magnetic field region in the northern hemisphere. Remarkably, the estimated resonant electron energies are much higher (up to $50 \mathrm{keV}$ ) than inferred from previous studies (up to $3 \mathrm{keV}$ ) for this kind of DAM emission, but compatible with in situ observations made by JADE and JEDI on the Juno spacecraft. Also, we quantitatively provided the explanation why a larger number of groups of arcs originating in the northern hemisphere are observed with respect to the ones originating in the southern hemisphere. The asymmetry of the magnetic field geometry and strength are the main responsible for this phenomenon.

\section{Acknowledgments, Samples, and Data}

This work is supported by the Juno Project under NASA grants NNM06AAa75c and $699041 X$ to SWRI and NASA grant NNN12AA01C to JPL/Caltech. The Juno mission is part of the New Frontiers Program managed at NASA's Marshall Space Flight Center in Huntsville, Alabama. Waves data used in this study can be found at the NASA Planetary Data System (PDS), https://pds.nasa.gov, under the dataset id: JNO-E_J_SS-WAV-3-CDR-SRVFULLV1.0. The specific Waves data displayed in Figure 2 are archived in Zenodo, https://doi.org/10.5281/zenodo.3884307. The JRM09 magnetic field model can be found in the Supporting Information of Connerney et al. [2018], https://doi.org/10.1002/2018GL077312. The VIP4 magnetic field model can be found in Table 1 of Connerney et al. [1998], https://doi.org/10.1029/97JA03726. Beaming cone half-angle outputs resulting from the forward modeling described in this study are located in the following link of Zenodo: https://doi.org/10.5281/zenodo.3884307.

\section{References}

Acuña, M. H., and Ness, N. F., (1976a), The main magnetic field of Jupiter. Journal of Geophysical Research, 81(16), 2917-2922.

Acuña, M. H., and Ness, N. F., (1976b), The magnetic field of Jupiter. In Magnetospheric particles and fields (pp. 311-323). Springer, Dordrecht.

Bagenal, F., Adriani, A., Allegrini, F., Bolton, S.J., Bonfond, B., Bunce, E.J., Connerney, J.E.P., Cowley, S.W.H., Ebert, R.W., Gladstone, G.R. and Hansen, C.J., (2017), Magnetospheric science objectives of the Juno mission. Space Science Reviews, 213(14), pp.219-287.

Bolton, S.J., Lunine, J., Stevenson, D., Connerney, J.E.P., Levin, S., Owen, T.C., Bagenal, F., Gautier, D., Ingersoll, A.P., Orton, G.S., Guillot, T., Hubbard, W., Bloxham, J., 
Coradini, A., Stephens, S.K., Mokashi, P., Thorne, R., and Thorpe, R., (2017), The Juno Mission. Space Science Reviews, 213(1-4), pp.5-37.

Bonfond, B., Hess, S., Gérard, J.C., Grodent, D., Radioti, A., Chantry, V., Saur, J., Jacobsen, S. and Clarke, J.T., (2013), Evolution of the Io footprint brightness I: Far-UV observations. Planetary and Space Science, 88, pp.64-75.

Burke, B.F. and Franklin, K.L., (1955), Observations of a variable radio source associated with the planet Jupiter. Journal of Geophysical Research, 60(2), pp.213-217.

Carr, T. D., M. D. Desch, and Alexander, J. K., (1983), Phenomenology of magnetospheric radio emissions, in Physics of the Jovian. Magnetosphere, edited by A. J. Dessler, pp. 226-284, Cambridge Univ. Press, New York

Clarke, J.T., Grodent, D., Cowley, S.W., Bunce, E.J., Zarka, P., Connerney, J.E. and Satoh, T. (2004), Jupiter's aurora. Jupiter: The Planet, Satellites and Magnetosphere, 1, pp.639670.

Connerney, J.E.P., Acuña, M.H. and Ness, N.F., (1981), Modeling the Jovian current sheet and inner magnetosphere. Journal Geophysical Research, 86, 8370 - 8384.

Connerney, E.P., (1992), Doing more with Jupiter's magnetic field in Planetary Radio Emissions III, edited by H.O. Rucker, S.J. Bauer, and M.L. Kaiser, pp. 13-33, Austrian Acad. Sci. press, Vienna.

Connerney, J. E. P., Acuña, M. H., Ness, N. F., and Satoh, T., (1998), New models of Jupiter's magnetic field constrained by the Io flux tube footprint. Journal of Geophysical Research, 103, 11,929-11,939. https://doi.org/10.1029/97JA03726

Connerney, J.E.P. and Satoh, T., (2000), The H3+ ion: A remote diagnostic of the Jovian magnetosphere. Philosophical Transactions of the Royal Society of London. Series A: Mathematical, Physical and Engineering Sciences, 358(1774), pp.2471-2483.

Connerney, J.E.P., Benn, M., Bjarno, J.B., Denver, T., Espley, J., Jorgensen, J.L., Jorgensen, P.S., Lawton, P., Malinnikova, A., Merayo, J.M., Murphy, S., Odom, J., Oliversen, R., Schnurr, R., Sheppard, D., Smith, E.J., (2017a), The Juno magnetic field investigation. Space Science Reviews, 213(1-4), pp.39-138.

Connerney, J.E., Adriani, A., Allegrini, F., Bagenal, F., Bolton, S.J., Bonfond, B., Cowley, S.W.H., Gerard, J.C., Gladstone, G.R., Grodent, D., Hospodarsky, G., Jorgensen, J. L., Kurth, W. S., Levin, S. M., Mauk, B., McComas, D. J., Mura, A., Paranicas, C., Smith, E. J., Thorne, R. M., Valek, P., Waite, J., (2017b), Jupiter's magnetosphere and aurorae observed by the Juno spacecraft during its first polar orbits. Science, 356(6340), pp.826-832.

Connerney, J.E.P., Kotsiaros, S., Oliversen, R.J., Espley, J.R., Jørgensen, J.L., Joergensen, P.S., Merayo, J.M., Herceg, M., Bloxham, J., Moore, K.M. and Bolton, S.J., (2018), A new model of Jupiter's magnetic field from Juno's first nine orbits. Geophysical Research Letters, 45(6), pp.2590-2596.

Ebert, R.W., Greathouse, T.K., Clark, G., Allegrini, F., Bagenal, F., Bolton, S.J., Connerney, J.E.P., Gladstone, G.R., Imai, M., Hue, V. and Kurth, W.S., (2019), Comparing Electron Energetics and UV Brightness in Jupiter's Northern Polar Region During Juno Perijove 5. Geophysical Research Letters.

Genova, F. and Aubier, M.G., (1985), Io-dependent sources of the Jovian decameter emission. Astronomy and Astrophysics, 150, pp.139-150. 
Galopeau, P. H. M., and M. Y. Boudjada, (2016), An oblate beaming cone for Io-controlled Jovian decameter emission, J. Geophys. Res. Space Physics, 121, 3120-3138, doi:10.1002/2015JA021038.

Gurnett, D.A. and Goertz, C.K., (1981). Multiple Alfven wave reflections excited by Io: Origin of the Jovian decametric arcs. Journal of Geophysical Research: Space Physics, 86(A2), pp.717-722.

Gurnett, D. A., and Bhattacharjee, A., (2005). Introduction to Plasma Physics With Space and Laboratory Applications. Cambridge, UK: Cambridge Univ. Press. https://doi.org/10.1017/CBO9780511809125

Hess, S., Cecconi, B., and Zarka, P., (2008), Modeling of Io-Jupiter decameter arcs, emission beaming and energy source. Geophysical Research Letters, 35(13).

Hess, S.L.G., Bonfond, B., Zarka, P. and Grodent, D., (2011), Model of the Jovian magnetic field topology constrained by the Io auroral emissions. Journal of Geophysical Research: Space Physics, 116(A5).

Hinton, P.C., Bagenal, F. and Bonfond, B., (2019), Alfvén Wave Propagation in the Io Plasma Torus. Geophysical Research Letters, 46, 1242-1249.

Hue, V., Greathouse, T. K., Bonfond, B., Saur, J., Gladstone, G. R., Roth, L., et al., (2019). Juno-UVS observation of the Io footprint during solar eclipse. Journal of Geophysical Research: Space Physics, 124, 5184- 5199. https://doi.org/10.1029/2018JA026431

Imai, M., K. Imai, C. A. Higgins, and J. R. Thieman, (2008), Angular beaming model of Jupiter's decametric radio emissions based on Cassini RPWS data analysis, Geophys. Res. Lett., 35, L17103, doi:10.1029/2008GL034987.

Imai, M., K. Imai, C. A. Higgins, and J. R. Thieman, (2011), Comparison between Cassini and Voyager observations of Jupiter's decametric and hectometric radio emissions, J. Geophys. Res., 116, A12233, doi:10.1029/2011JA016456.

Imai, M., Kurth, W.S., Hospodarsky, G.B., Bolton, S.J., Connerney, J.E., Levin, S.M., Lecacheux, A., Lamy, L. and Zarka, P., (2017), Latitudinal beaming of Jovian decametric radio emissions as viewed from Juno and the Nançay Decameter Array. Geophysical Research Letters, 44(10), pp.4455-4462.

Kaiser, M. L., Zarka, P., Kurth, W. S., Hospodarsky, G. B. and Gurnett, D. A., (2000), Cassini and Wind stereoscopic observations of Jovian nonthermal radio emissions: Measurement of beam widths, Journal of Geophysical Research, 105, 16,053-16,062.

Kotsiaros, S., Connerney, J.E., Clark, G., Allegrini, F., Gladstone, G.R., Kurth, W.S., Mauk, B.H., Saur, J., Bunce, E.J., Gershman, D.J., Martos, Y.M., Greathouse, T. K., Bolton, S. J., and Levin, S. M., (2019), Birkeland currents in Jupiter's magnetosphere observed by the polar-orbiting Juno spacecraft. Nature Astronomy, 3(10), pp.904-909.

Kurth, W.S., Imai, M., Hospodarsky, G.B., Gurnett, D.A., Louarn, P., Valek, P., Allegrini, F., Connerney, J.E.P., Mauk, B.H., Bolton, S.J. and Levin, S.M., (2017a), A new view of Jupiter's auroral radio spectrum. Geophysical Research Letters, 44(14), pp.7114-7121.

Kurth, W.S., Hospodarsky, G.B., Kirchner, D.L., Mokrzycki, B.T., Averkamp, T.F., Robison, W.T., Piker, C.W., Sampl, M. and Zarka, P., (2017b), The Juno waves investigation. Space Science Reviews, 213(1-4), pp.347-392.

Louis, C.K., Lamy, L., Zarka, P., Cecconi, B., Imai, M., Kurth, W.S., Hospodarsky, G., Hess, S.L.G., Bonnin, X., Bolton, S. and Connerney, J.E.P., (2017), Io-Jupiter decametric arcs 
observed by Juno/Waves compared to ExPRES simulations. Geophysical Research Letters, 44(18), pp.9225-9232.

Louis, C.K., Hess, S.L.G., Cecconi, B., Zarka, P., Lamy, L., Aicardi, S. and Loh, A., (2019), ExPRES: an Exoplanetary and Planetary Radio Emissions Simulator. Astronomy \& Astrophysics, 627, p.A30.

Louarn, P., Allegrini, F., McComas, D.J., Valek, P.W., Kurth, W.S., André, N., Bagenal, F., Bolton, S., Connerney, J., Ebert, R.W. and Imai, M., (2017), Generation of the Jovian hectometric radiation: First lessons from Juno. Geophysical Research Letters, 44(10), pp.4439-4446.

Louarn, P., Allegrini, F., McComas, D. J., Valek, P. W., Kurth, W. S., André, N., et al., (2018). Observation of electron conics by Juno: Implications for radio generation and acceleration processes. Geophysical Research Letters, 45, 9408-9416. https://doi.org/10.1029/2018GL078973

Mauk, B.H., Haggerty, D.K., Jaskulek, S.E., Schlemm, C.E., Brown, L.E., Cooper, S.A., Gurnee, R.S., Hammock, C.M., Hayes, J.R., Ho, G.C. and Hutcheson, J.C., (2017). The Jupiter energetic particle detector instrument (JEDI) investigation for the Juno mission. Space Science Reviews, 213(1-4), pp.289-346.

Marques, M.S., Zarka, P., Echer, E., Ryabov, V.B., Alves, M.V., Denis, L. and Coffre, A., (2017), Statistical analysis of $26 \mathrm{yr}$ of observations of decametric radio emissions from Jupiter. Astronomy \& Astrophysics, 604, p.A17.

McComas, D.J., Alexander, N., Allegrini, F., Bagenal, F., Beebe, C., Clark, G., Crary, F., Desai, M.I., De Los Santos, A., Demkee, D. and Dickinson, J., (2017), The Jovian auroral distributions experiment (JADE) on the Juno Mission to Jupiter. Space Science Reviews, 213(1-4), pp.547-643.

Mura, A., Adriani, A., Connerney, J.E.P., Bolton, S., Altieri, F., Bagenal, F., Bonfond, B., Dinelli, B.M., Gérard, J.C., Greathouse, T., Grodent, D., et al., (2018), Juno observations of spot structures and a split tail in Io-induced aurorae on Jupiter. Science, 361(6404), pp.774-777.

Panchenko, M. and Rucker, H.O., (2016), Estimation of emission cone wall thickness of Jupiter's decametric radio emission using stereoscopic STEREO/WAVES observations. Astronomy \& Astrophysics, 596, p.A18.

Queinnec, J., and Zarka, P., (1998), Io-controlled decameter arcs and Io-Jupiter interaction, J. Geophys. Res., 103, 26,649-26,666, doi:10.1029/98JA02435.

Ray, L. C., and Hess, S., (2008), Modelling the Io-related DAM emission by modifying the beaming angle. Journal of Geophysical Research: Space Physics, 113(A11).

Szalay, J.R., Bonfond, B., Allegrini, F., Bagenal, F., Bolton, S., Clark, G., Connerney, J.E.P., Ebert, R.W., Ergun, R.E., Gladstone, G.R. and Grodent, D., (2018), In Situ Observations Connected to the Io Footprint Tail Aurora. Journal of Geophysical Research: Planets, 123(11), pp.3061-3077.

Thomas, N., Bagenal, F., Hill, T.W. and Wilson, J.K., (2004), The Io neutral clouds and plasma torus. Jupiter. The planet, satellites and magnetosphere, 1, pp.561-591.

Treumann, R. A., (2006), The electron-cyclotron maser for astrophysical application, Astron. Astrophys. Rev., 13(4), 229-315, doi:10.1007/s00159-006-0001-y. 
Wilkinson, M.H., (1989), Io-related Jovian decametric arcs. Journal of Geophysical Research: Space Physics, 94(A9), pp.11777-11790.

Wu, C. S., and Lee, L. C., (1979), A theory of the terrestrial kilometric radiation, Astrophys. J., 230, 621- 626 .

Zarka, P., (1998), Auroral radio emissions at the outer planets: Observations and theories. Journal of Geophysical Research: Planets, 103(E9), pp.20159-20194.

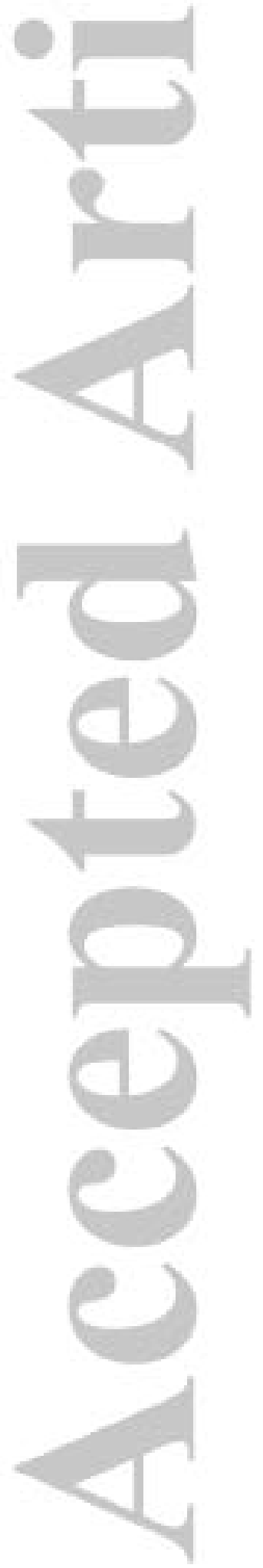




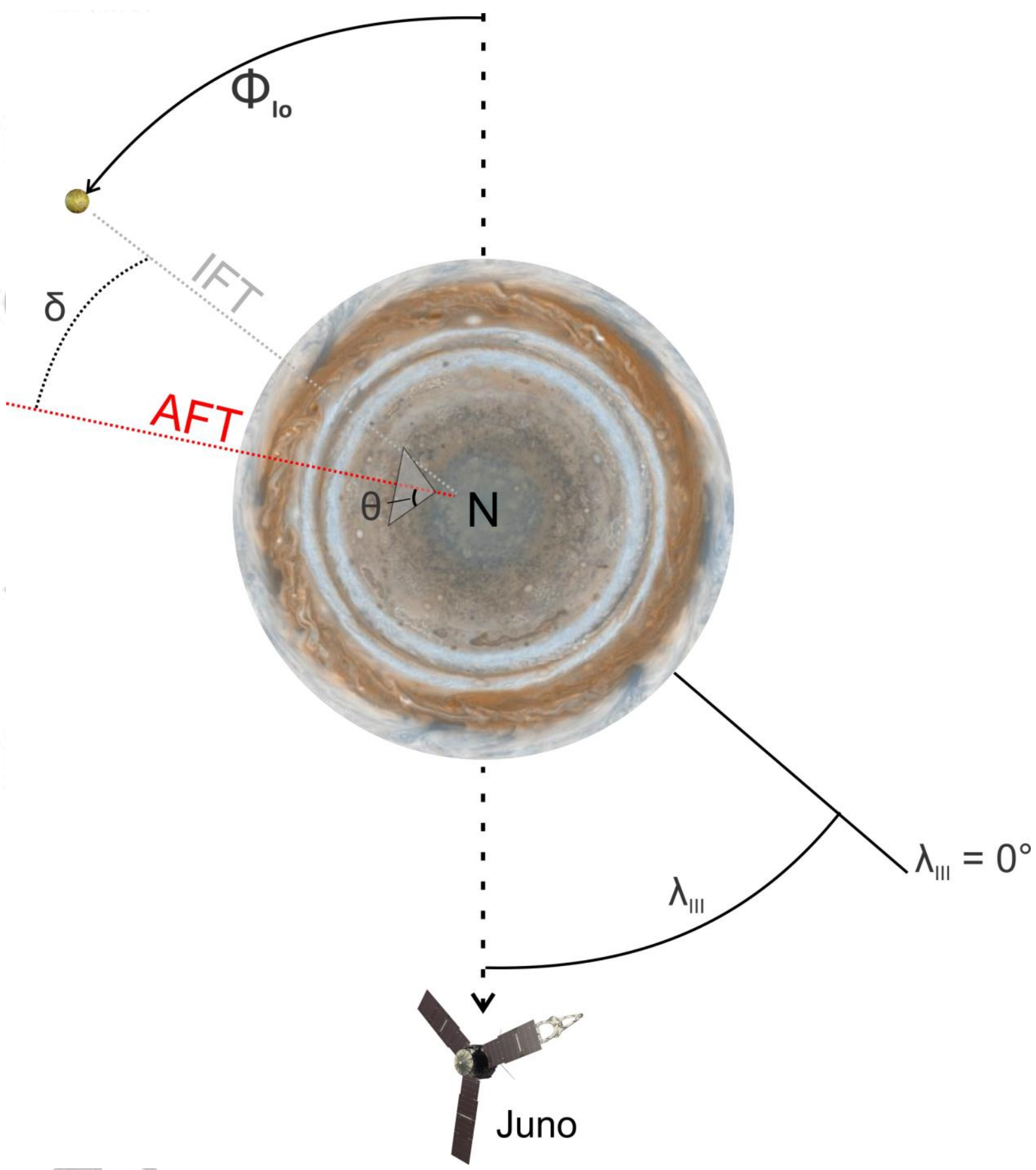

Figure 1. North plan view of the Jupiter-Io-Juno geometrical configuration. $\Phi_{\text {Io }}$ denotes Io phase. $\lambda_{\text {III }}$ is the longitude of the observer (Juno) or Central Meridian Longitude (CML) in the System III coordinate system. $\delta$ represents the lead angle. $\Theta$ is the cone half-angle beaming angle. IFT: Io Flux Tube, AFT: Active Flux Tube. 

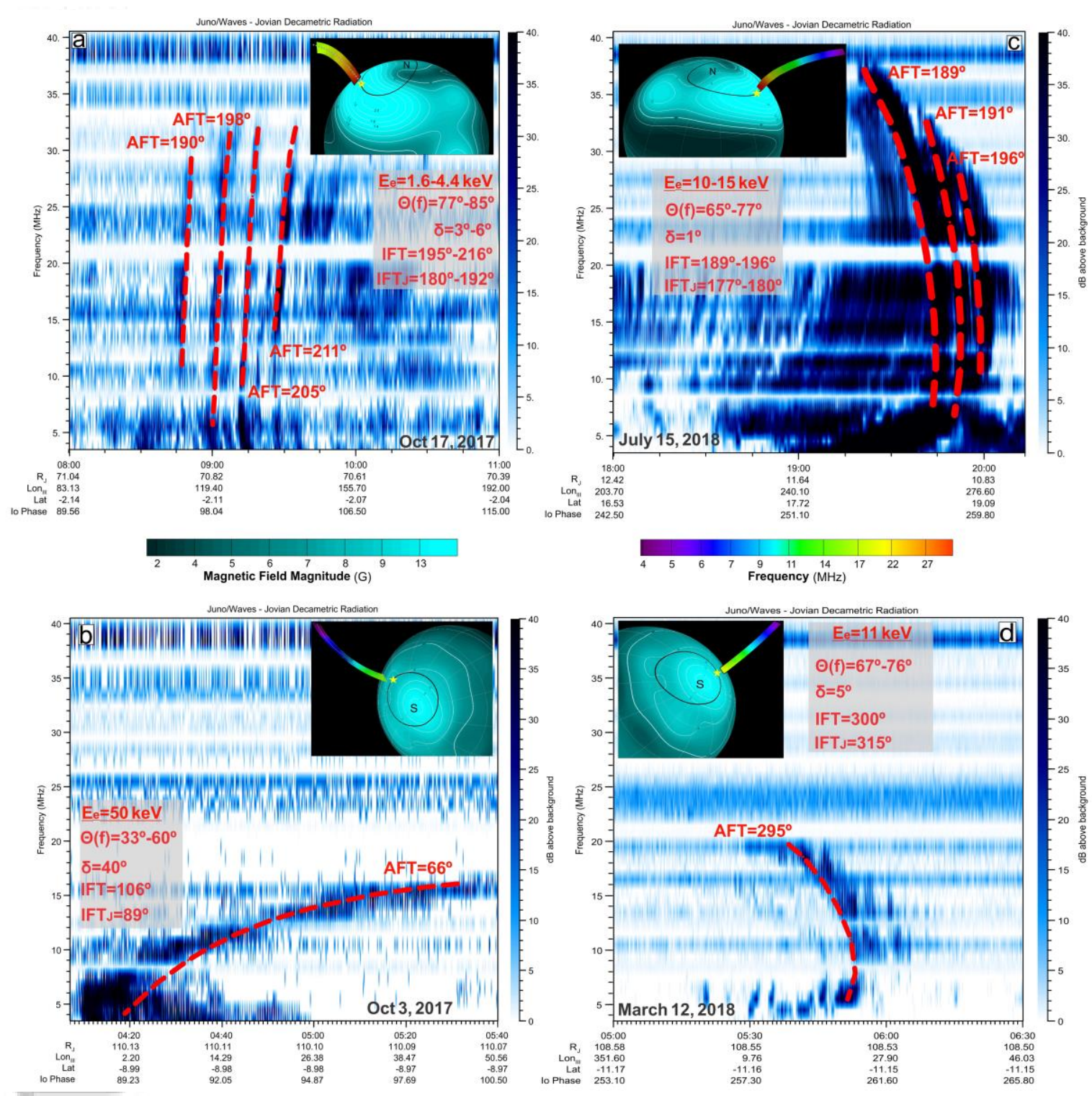

Figure 2. Decametric radio emissions observed by Juno and their forward models. Panels a and $\mathrm{b}$ correspond to vertex early emissions and $\mathrm{c}$ and $\mathrm{d}$ to vertex late emissions. The observations shown in panels $\mathrm{a}$ and $\mathrm{b}$ originate in the northern hemisphere while the ones on panels $\mathrm{c}$ and $\mathrm{d}$ are from the southern hemisphere. a) Discrete arcs originating in the B region. b) Arc originating in the D source region. c) Discrete arcs from the A region. d) Arc from the $\mathrm{C}$ region. $\mathrm{E}_{\mathrm{e}}$ denotes the estimated energy for the resonant electrons responsible for the emissions. AFT: Active Flux Tube, IFT: Io Flux Tube at Io orbit, IFT,: projection of the IFT at Jupiter's surface, $\delta$ : lead angle, $\Theta$ (f) is the beaming cone half-angle which depends on the frequency of the emission. The insets included in the panels illustrate the location of the radio sources responsible for the observed arcs and the foot print of the magnetic field line that carries the radio sources on the planet's surface. The magnetic field magnitude predicted by the JRM09 magnetic field model [Connerney et al., 2018] is displayed on the surface of the planet. The black circular line represents the Io foot print predicted by JRM09 on Jupiter's surface. Lon III is the Central Meridian Longitude or longitude of Juno referred to as the System III coordinate system, $\lambda_{\text {III }}$ in Figure 1.

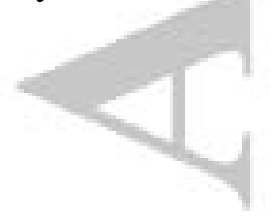




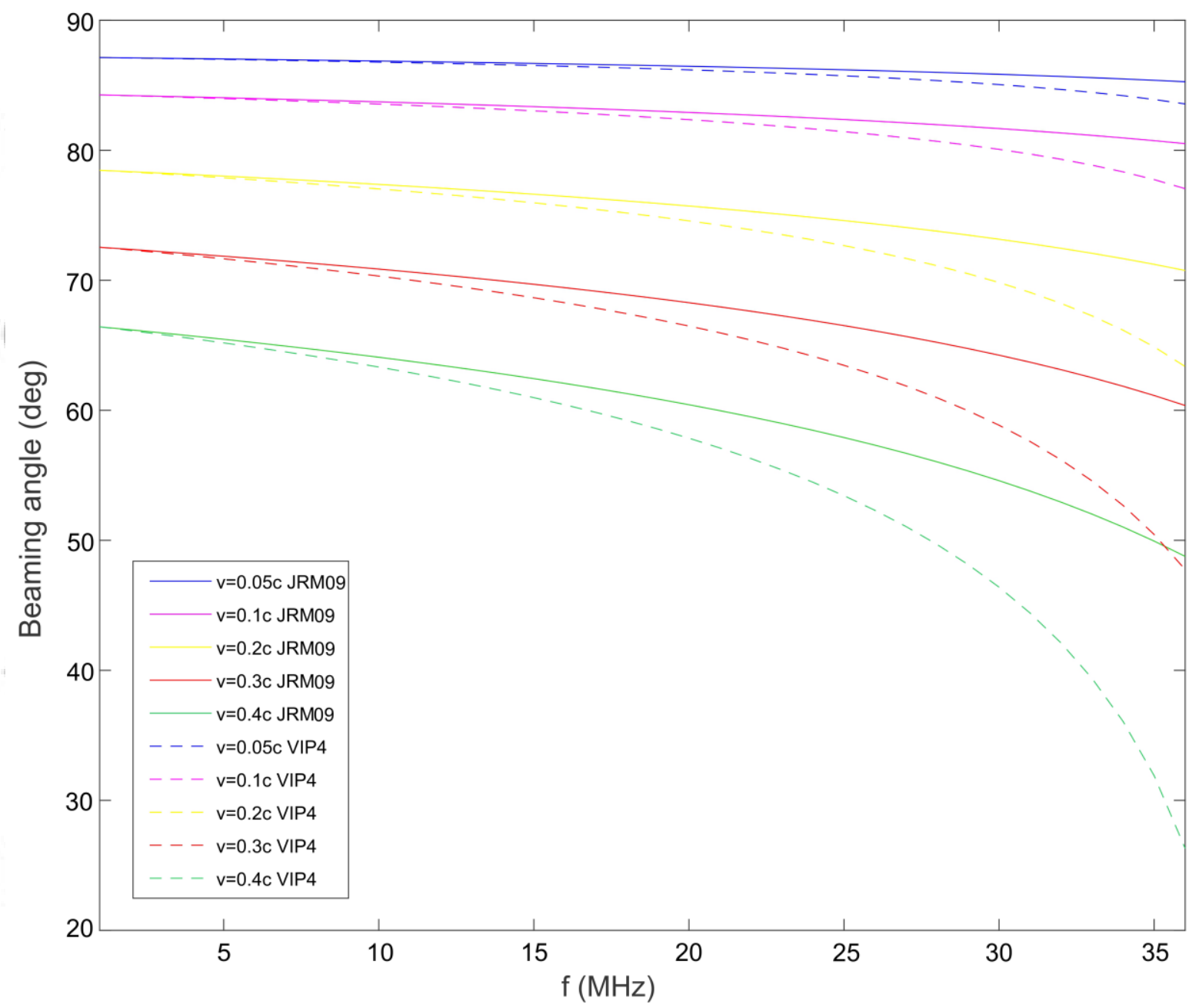

Figure 3. Relationship between the cone half-angle and observed frequency assuming a losscone instability (equation 1), and the JRM09 and the VIP4 magnetic field models. The maximum possible frequency in equation 1 is taken from the highest gyrofrequency predicted at the Io footprint by each model, which is located in the northern hemisphere of Jupiter. The curves are representing the beaming angle solutions considering energies for the resonant electrons of $0.64\left(\mathrm{v}_{\mathrm{e}}=0.05 \mathrm{c}\right), 2.5\left(\mathrm{v}_{\mathrm{e}}=0.1 \mathrm{c}\right), 10.2 \quad\left(\mathrm{v}_{\mathrm{e}}=0.2 \mathrm{c}\right), 23\left(\mathrm{v}_{\mathrm{e}}=0.3 \mathrm{c}\right)$, and $40.8 \mathrm{keV}$ $\left(v_{e}=0.4 c\right)$. The use of the VIP4 fails to yield realistic solutions for electron energies above 40 $\mathrm{keV}\left(\mathrm{v}_{\mathrm{e}}=0.4 \mathrm{c}\right)$ and for local frequencies higher than $36 \mathrm{MHz}$. 

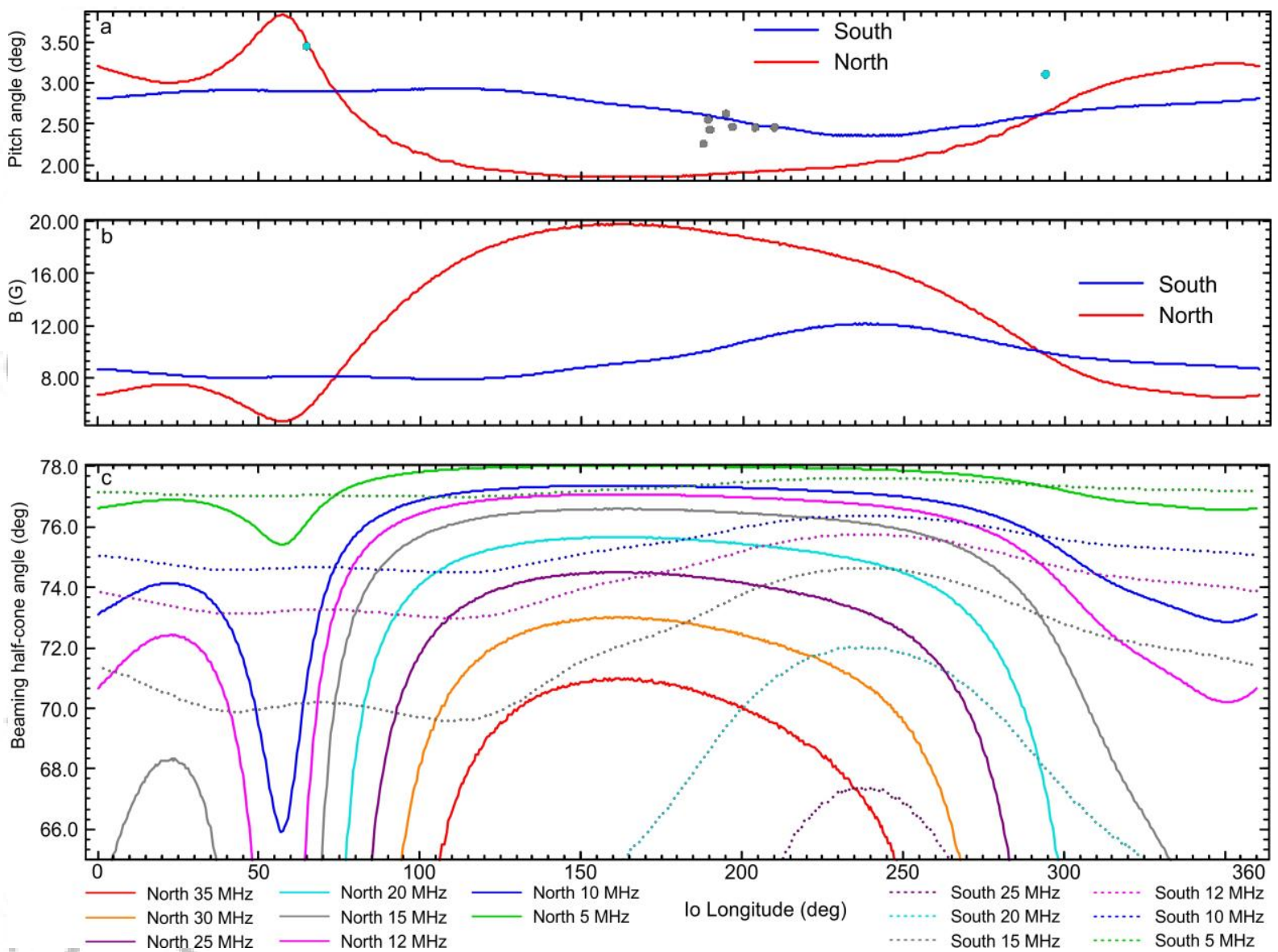

Figure 4. Magnetic field and pitch angles. a) Equatorial pitch angles at Io's orbit and considering mirror points at the Io foot print. Dots represent the calculated pitch angles for modeled arcs (grey for A and B, and blue for C and D) for which the peak frequencies have been taken as the mirror point. b) is the magnetic field magnitude at the Io foot print. c) Beaming cone half-angle as a function of the local gyrofrequency. Note the constant values found for the northern hemisphere and centered in $\sim 180^{\circ}$. See text for details. 


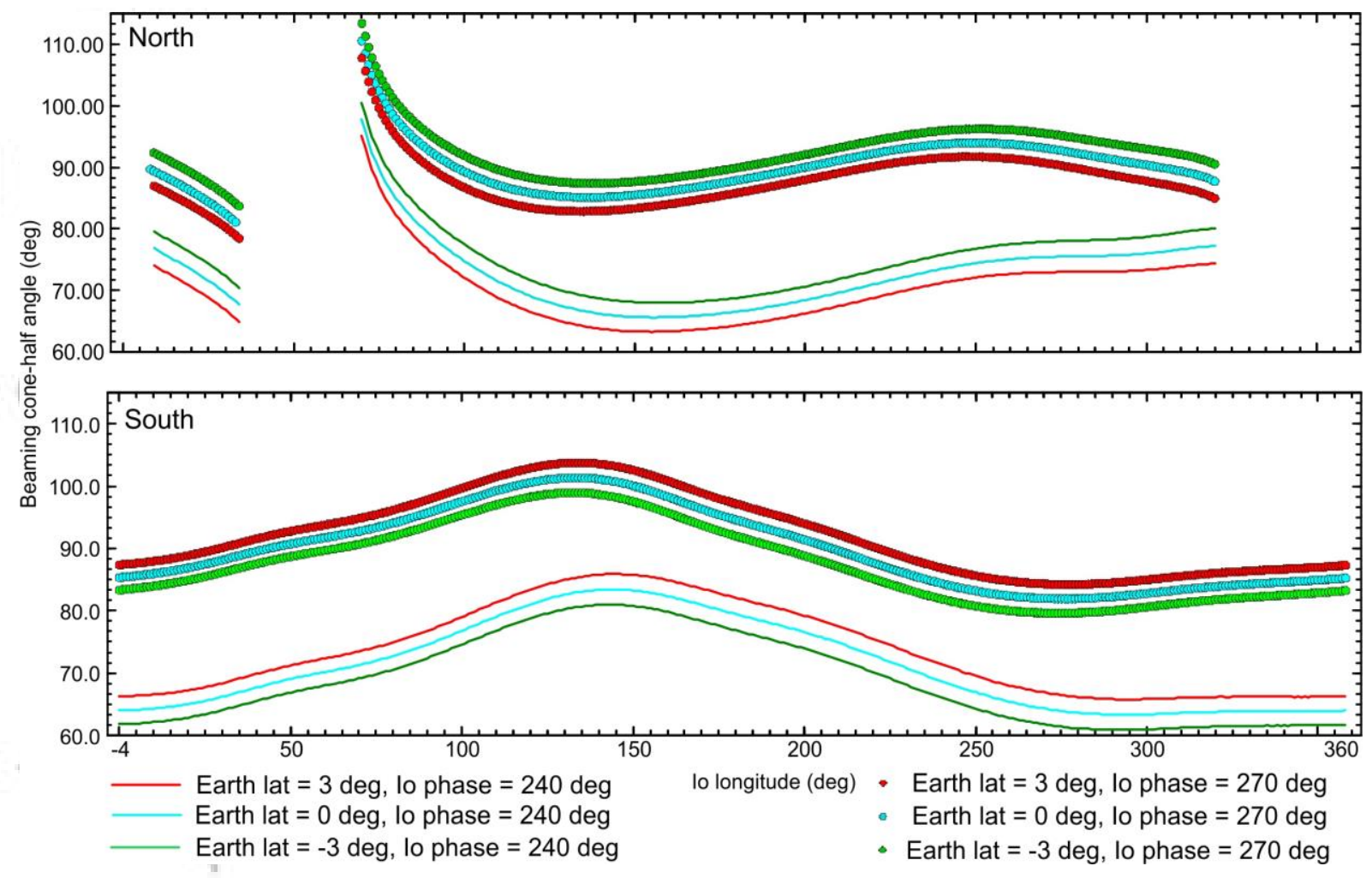

Figure 5. Beaming cone half-angle values for a radio source of $20 \mathrm{MHz}$ and a fixed geometry between Earth (at $5 \mathrm{AU}$ ) and Io in vertex late configuration, with a rotating Jupiter to investigate the influence of the asymmetrical geometry of the magnetic field into the observability of DAM. Earth's latitude varies between -3 and $3^{\circ}$. The northern hemisphere generally shows less variable beaming cone half-angles from one AFT (or Jovian longitude) to the next than the southern hemisphere. Note that the beaming cone half-angles shown in this graph for the southern hemisphere refer to the acute angle between the radio wave and the magnetic field vector. See text for details. 